\section{Pediatric thioridazine poisoning as a result of a pharmacy compounding error}

\author{
Zenichiro Kato, ${ }^{1-4}$ Mitsuhiro Nakamura, ${ }^{5}$ \\ Yuka Yamagishi,' Takahide Teramoto, \\ Naomi Kondo ${ }^{1-3}$
}

'Department of Pediatrics, Graduate School of Medicine, ${ }^{2}$ Center for Emerging Infectious Diseases, and ${ }^{3}$ Center for Advanced Drug Research, Gifu University, Gifu, Japan; ${ }^{4}$ Molecular Cellular Biology, Harvard University, Cambridge MA, USA; ${ }^{5}$ Laboratory of Drug Informatics, Gifu Pharmaceutical University, Gifu, Japan

\section{Abstract}

The adverse effects or overdose of thioridazine including sudden death, fatal arrhythmia, or retinopathy, in addition to the neurological signs have been reported. A three-yearold boy with bronchitis was prescribed erythromycin by a local clinic, but he started to complain of severe drowsiness and became unconscious. It was decided that this was a result of a compounding error of thioridazine instead of erythromycin owing to their similar commercial names. The thioridazine concentration in the child's serum on admission was two to three times higher than the Cmax for adults with the same dosage. The concentration of the lavage saline on admission was only $0.3 \%$ of the ingested amount, indicating that the lavage was not effective in our case. Pharmacokinetic analysis revealed the parameters as Tmax, $1.5 \mathrm{hr}$; Cmax, $1700 \mathrm{ng} / \mathrm{mL}$; Ka $2.01 \mathrm{~L} / \mathrm{hr} ; \mathrm{Vd}, 3.6 \mathrm{~L} / \mathrm{kg}$; and T1/2, $6.8 \mathrm{hr}$. Further investigations on clinical cases with a pharmacokinetic analysis should be done to confirm the pharmacokinetic evidence obtained here and to give specific therapeutic guidelines for overdose management especially in children.

\section{Introduction}

Thioridazine is a phenotiazine with potent anxiolytic and antipsychotic properties. ${ }^{1}$ Recently the adverse effects or overdose of thioridazine including sudden death, fatal arrhythmia, retinopathy, and neurological signs and symptoms have been reported. ${ }^{1.3} \mathrm{We}$ describe here a case of pediatric thioridazine toxicity caused by a pharmacy compounding error.

\section{Case Report}

On the morning of presentation to our department a three-year-old boy with bronchitis was prescribed erythromycin by a local clinic. The child took one dose of the compounded drug at the pharmacy at about 13:00, after which he started to complain of sleepiness and then became unconscious. His mother called the pharmacy and it was noticed that there was a compounding error of thioridazine instead of erythromycin owing to their similar commercial names. The prescribed drug for the patient was one-gram of powder per dose (100 mg of thioridazine per dose). The child was transferred to our hospital and admitted to the pediatric unit.

On admission he was unconsciousness with E3, V4, M5 of the Glasgow coma scale. A gastric lavage was performed and fluid infusion with intensive monitoring was started. The family history and the past medical history were unremarkable. A physical examination revealed a temperature of $36.9^{\circ} \mathrm{C}$, blood pressure of $119 / 56 \mathrm{mmHg}$, pulse of 148 beats/minute, respiratory rate of 40 breaths $/ \mathrm{min}$, and weight of $13 \mathrm{~kg}$. A neurological examination revealed normal eye movements without nystagmus, but extensive drowsiness. His pulse oximetry reading was $100 \%$. An electrocardiogram was negative for dysrhythmias and elongation QT intervals. The laboratory investigation including a hemogram, serum electrolytes, calcium, phosphorus, liver function tests, and renal function tests was normal. A brain computed tomography (CT) scan showed mild diffuse edema. We used $100 \mathrm{~mL}$ of $10 \%$ glycerol and 10 mg of flosemide. He developed no other severe symptoms, and he awoke 18 hours after the dosage but felt mildly sleepy for several hours. The follow-up ECG and brain CT did not show any abnormality on the fifth day after admission. He required no further pharmacological intervention for the intoxication, but was treated for bronchitis for the following four days and was discharged in a stable condition.

The thioridazine concentrations of the specimens were determined by high performance liquid chromatography (HPLC) compared with
Correspondence: Zenichiro Kato, Department of Pediatrics, Graduate School of Medicine, Gifu University, Yanagido 1-1, Gifu 501-1194, Japan. E-mail: zen-k@gifu-u.ac.jp

Key words: thioridazine, compounding error, pharmacokinetics.

Conflict of interest: the authors report no conflicts of interest.

Received for publication: 20 June 2009 Revision received: 7 November 2009.

Accepted for publication: 9 November 2009 .

This work is licensed under a Creative Commons Attribution 3.0 License (by-nc 3.0).

(C) Copyright Z. Kato et al., 2009

Licensee PAGEPress, Italy

Pediatric Reports 2009; 1:e9

doi:10.4081/pr.2009.e9

a standard thioridazine preparation of known concentration (Sigma Chemical, St. Louis, MO, USA) using specimens of serum, urine, and lavage saline. The results showed the presence of a high amount of thioridazine. The thioridazine concentrations were 1124.5 $\mathrm{ng} / \mathrm{mL}$ in the serum on admission, $20.2 \mathrm{ng} / \mathrm{mL}$ in the first urine sample after admission, and $1625.8 \mathrm{ng} / \mathrm{mL}$ in $200 \mathrm{~mL}$ of the lavage saline on admission. On the other hand the thioridazine concentrations were $277.6 \mathrm{ng} / \mathrm{mL}$, and were not detected in the serum on the next day and three days later. Pharmacokinetic analysis revealed the parameters as Tmax, $1.5 \mathrm{hr}$; Cmax, 1700 ng/mL; Ka, 2.01 L/hr; Vd, 3.6 L/kg; and T1/2, $6.8 \mathrm{hr}$ (Figure 1).

\section{Discussion}

Thioridazine usage in childhood is rare and the information about its pharmacology and pharmacokinetics is recognized rarely among pediatricians. ${ }^{1.3}$ The most frequent adverse effects of thioridazine observed in 159 cases

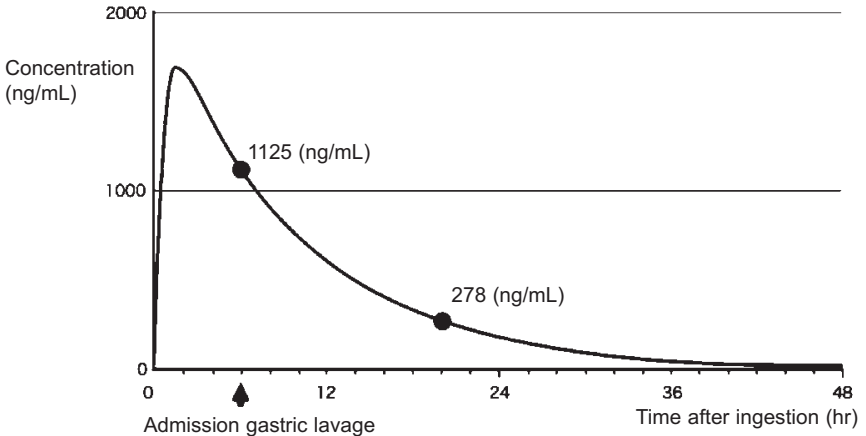

Figure 1. Pharmacokinetic analysis of thioridazine concentration. Closed circles indicate the observed values in the serum. 
were impairment of consciousness (98/159), arrhythmia and/or ECG changes (68/159), extrapyramidal symptoms (26/159), confusion (22/159), agitation (19/159), and respiratory disorders (13/159). The correlation between the dosage and the degree of cardiovascular adverse effects was observed. ${ }^{1}$ However, no information about the serum concentrations in these cases was provided. Attention should be focused on the management of the cardiovascular disturbances because of their fatal outcome.

The recommended therapeutic approaches for the overdose of thioridazine are gastric lavage, administration of activated charcoal to eliminate the drug, and securing an open airway in unconscious patients. The solution from the gastric lavage at six hours after ingestion had a concentration of $1625.8 \mathrm{ng} / \mathrm{mL}$, which is a total of $325 \mu \mathrm{g}$ in $200 \mathrm{~mL}$ saline. This total amount is only $0.3 \%$ of the ingested amount indicating that the lavage was not effective in our case possibly because the absorption phase was completed, as indicated in Figure 1. Although we could not measure the sulfoxide and sulfonated metabolites of thioridazine, they have similar effects to the thioridazine itself and their elimination from the body is prolonged. ${ }^{4,5}$

The values obtained from the adults who took $200 \mathrm{mg}$ of the drug showed the values as Tmax, $3.3 \mathrm{hr}$; Cmax, $750 \mathrm{ng} / \mathrm{mL}$; Ka, $2.11 \mathrm{~L} / \mathrm{hr}$; Vd, 3.5 $\mathrm{L} / \mathrm{kg}$; and T1/2, $7.5 \mathrm{hr}$; indicating that the pharmacokinetics in children generally can be treated as those in the adults, except for the absorption phase. ${ }^{4,5}$ However, Cmax should be much higher than those of the adults owing to the higher dosage per body weight, having a high risk for adverse effects. ${ }^{46}$ In addition the lavage may not be effective after one hour possibly because of its quick absorption kinetics in infants compared to that in adults. Further investigations on clinical cases with a pharmacokinetic analysis should be done to confirm the pharmacokinetic evidence obtained in our case and to give specific therapeutic guidelines for overdose management especially in children.

\section{References}

1. Le Blaye I, Donatini B, Hall M, et al. Acute overdosage with thioridazine: a review of the available clinical exposure. Vet Hum Toxicol 1993;35:147-50.

2. Copetti R, Proclemer A, Pillinini PP. Brugada-like ECG abnormalities during thioridazine overdose. Br J Clin Pharmacol 2005;59:608.

3. Salih IS, Thanacoody RH, McKay GA, et al. Comparison of the effects of thioridazine and mesoridazine on the QT interval in healthy adults after single oral doses. Clin Pharmacol Ther 2007;82:548-54.

4. Vanderheeren FA, Muusze RG. Plasma levels and half lives of thioridazine and some of its metabolites. I. High doses in young acute schizophrenics. Eur J Clin Pharmacol 1977;11:135-40.

5. Chakraborty BS, Midha KK, McKay G, et al. Single dose kinetics of thioridazine and its two psychoactive metabolites in healthy humans: a dose proportionality study. J Pharm Sci 1989;78:796-801.

6. Hartigan-Go K, Bateman DN, Nyberg G, et al. Concentration-related pharmacodynamic effects of thioridazine and its metabolites in humans. Clin Pharmacol Ther 1996;60:543-53. 